\title{
Novel T-violation observable open to any decay channel at meson factories
}

\author{
José Bernabéu \\ Departament de Física Teòrica and IFIC, Universitat de València - CSIC \\ E-46100, Burjassot, Spain. \\ E-mail: jose.bernabeuluv.es \\ Francisco J. Botella ${ }^{1}$ \\ Departament de Física Teòrica and IFIC, Universitat de València - CSIC \\ E-46100, Burjassot, Spain. \\ E-mail: francisco.botelladific.uv.es
}

\section{Miguel Nebot}

Centro de Física Teórica de Partículas-CFTP, Instituto Superior Técnico-IST, Universidade de Lisboa-UL Avenida Rovisco Pais, 1049-001 Lisboa, Portugal.

E-mail: miguel. nebotauv.es

\begin{abstract}
Quantum entanglement between the two neutral mesons produced in meson factories has allowed the first direct observation of Time Reversal Violation in the time evolution of the B neutral meson between the two decays. The exceptional meson transitions are directly connected to semileptonic and CP eigenstate decay channels. The possibility of extending the observable asymmetries to more decay channels confronts the problem of the "orthogonality condition": Given a decay channel $f$, which is the decay channel $f$ ' such that the meson state not decaying to $f$ ' is orthogonal to the meson state not decaying to $f$ ? We have proposed an alternative TViolation asymmetry at meson factories which allows its opening to any pair of decay channels. Instead of searching which is the pair of decay channels associated to the T-reverse meson transition, we build an asymmetry which tags the initial states of both the Reference and the Treverse meson transitions. This observable filters the appropriate final states by means of two measurable survival probabilities. We discuss the methodology to be followed in the analysis of the new observable and the results expected in specific examples.
\end{abstract}

Proceedings of the Corfu Summer Institute 2014 "School and Workshops on Elementary Particle Physics and Gravity"

3-21 September 2014

Corfu, Greece

\section{${ }^{1}$ Speaker}




\section{Introduction}

The BABAR Collaboration has recently reported, in the $B^{0}-\bar{B}^{0}$ system, the first direct observation of T-violation [1], in the time evolution of any system with high statistical significance. The measurement is based in a method described in [2] following the concepts originally proposed in $[3,4]$. In the $\left\{\left|P^{0}\right\rangle\left|\bar{P}^{0}\right\rangle\right\}$ system, where $P^{0}$ stands for a neutral meson $K^{0}, D^{0}, B_{d}^{0}$ or $B_{S}^{0}$ (and $\bar{P}^{0}$ for the corresponding antimeson), we define two arbitrary states

$$
\begin{aligned}
& \left|M_{1}\right\rangle=m_{1}{ }^{0}\left|P^{0}\right\rangle+\bar{m}_{1}{ }^{0}\left|\bar{P}^{0}\right\rangle \\
& \left|M_{2}\right\rangle=m_{2}{ }^{0}\left|P^{0}\right\rangle+\bar{m}_{2}{ }^{0}\left|\bar{P}^{0}\right\rangle
\end{aligned}
$$

The T-violation observable is then given by

where

$$
A_{12}(t)=\frac{\operatorname{Pr}\left[M_{1} \rightarrow M_{2}(t)\right]-\operatorname{Pr}\left[M_{2} \rightarrow M_{1}(t)\right]}{\operatorname{Pr}\left[M_{1} \rightarrow M_{2}(t)\right]+\operatorname{Pr}\left[M_{2} \rightarrow M_{1}(t)\right]}
$$

$$
\operatorname{Pr}\left[M_{1} \rightarrow M_{2}(t)\right]=\left|\left\langle M_{2} \mid M_{1}(t)\right\rangle\right|^{2}=\left|\left\langle M_{2}|U(t, 0)| M_{1}\right\rangle\right|^{2}
$$

is the probability that an initially prepared state $M_{1}$, evolving after time $t$ to $M_{1}(t)$, behave like state $M_{2}$. The BABAR asymmetry is build from $M_{1}=B_{d}^{0}, \bar{B}_{d}^{0}$ and $M_{2}=B_{ \pm}$where $B_{ \pm}$are the $B$-states tagged or filtered by the decays to CP-eigenstates with definite flavour content. These asymmetries are experimentally independent of CP violation. The Kabir asymmetry [5], with $M_{1}, M_{2}=K^{0}, \bar{K}^{0}$ was measured by the CPLEAR Collaboration [6] with a non-vanishing value near 4 standard deviations.

\section{The Reference Transition at $\mathrm{C}=-$ initial entangled}

The two quantum effects:

- Entanglement between the two neutral mesons produced at a meson factory

- And the filtering measurement induced by the meson decay are essential ingredients to be incorporated in the analysis for the preparation of initial and final meson states. We want to find out which is the Reference Transition $M_{1} \rightarrow$ $M_{2}(t)$ between meson states - some particular combination of $P^{0}$ and $\bar{P}^{0}$ - associated to a given pair of decays: $f_{1}$ at $t_{1}$ and $f_{2}$ at ${ }_{2}>t_{1}$, here denoted by $\left(f_{1}, f_{2}\right)$.

The entangled state of the two mesons in an antisymmetric combination of individual orthogonal states,

$$
\left|\Phi_{(C=-)}\right\rangle=\frac{1}{\sqrt{2}}\left\{\left|P^{0}(\vec{k})\right\rangle\left|\bar{P}^{0}(-\vec{k})\right\rangle-\left|\bar{P}^{0}(\vec{k})\right\rangle\left|P^{0}(-\vec{k})\right\rangle\right\}
$$

tells us that the (still living) meson at time $t_{1}$ is tagged as "the state that does not decay into $f^{\prime \prime}[7],[8]$

$$
\left|P_{\nrightarrow f}\right\rangle=\frac{1}{\sqrt{\left(\left|A_{f}\right|^{2}+\left|\bar{A}_{f}\right|^{2}\right)}}\left\{\bar{A}_{f}\left|P^{0}\right\rangle-A_{f}\left|\bar{P}^{0}\right\rangle\right\}
$$

where $A_{f}\left(\bar{A}_{f}\right)$ is the decay amplitude from $P^{0}\left(\bar{P}^{0}\right)$ to $f$ : 


$$
A_{f}=\left\langle f|W| P^{0}\right\rangle ; \bar{A}_{f}=\left\langle f|W| \bar{P}^{0}\right\rangle
$$

to first order in the weak Hamiltonian $H_{w}$ and to all orders in strong interactions $W=U_{S}(\infty, 0) H_{w} . U_{S}(\infty, 0)$ is the strong evolution operator and is equal to the identity if we can neglect final sate interactions. (For hadronic decays we will assume transitions with one helicity amplitude: $0 \rightarrow 0+j$ ).

The corresponding orthogonal state $\left\langle P_{\rightarrow f}^{\perp} \mid P_{\rightarrow f}\right\rangle=0$ is given by

$$
\left|P_{\rightarrow f}^{\perp}\right\rangle=\frac{1}{\sqrt{\left(\left|A_{f}\right|^{2}+\left|\bar{A}_{f}\right|^{2}\right)}}\left\{A_{f}^{*}\left|P^{0}\right\rangle+\bar{A}_{f}^{*}\left|\bar{P}^{0}\right\rangle\right\}
$$

and it is the one filtered by the decay. What we call the "filtering identity" defines the precise meaning of this statement [9]:

Note that we can also write

$$
\left|\left\langle P_{\rightarrow f_{2}}^{\perp} \mid M_{1}\right\rangle\right|^{2}=\frac{\left|\left\langle f_{2}|W| M_{1}\right\rangle\right|^{2}}{\left(\left|A_{f_{2}}\right|^{2}+\left|\bar{A}_{f_{2}}\right|^{2}\right)}
$$

$$
\begin{gathered}
\left|\Phi_{(C=-)}\right\rangle=\frac{1}{\sqrt{2}}\left\{\left|P^{0}(\vec{k})\right\rangle\left|\bar{P}^{0}(-\vec{k})\right\rangle-\left|\bar{P}^{0}(\vec{k})\right\rangle\left|P^{0}(-\vec{k})\right\rangle\right\}= \\
=\frac{1}{\sqrt{2}}\left\{\left|P_{\nrightarrow f}^{\perp}(\vec{k})\right\rangle\left|P_{\nrightarrow f}(-\vec{k})\right\rangle-\left|P_{\nrightarrow f}(\vec{k})\right\rangle\left|P_{\nrightarrow f}^{\perp}(-\vec{k})\right\rangle\right\}
\end{gathered}
$$

Experimentally, the Reference Transition $M_{1} \rightarrow M_{2}(t)$ is therefore directly connected to ${ }_{1}=P_{\rightarrow f_{1}}, M_{2}=P_{\rightarrow f_{2}}^{\perp}$, i.e.,

$$
P_{\rightarrow f_{1}}\left(t_{1}\right) \rightarrow P_{\rightarrow f_{2}}^{\perp}\left(t_{2}\right)
$$

The $T$ transformed transition

$$
P_{\rightarrow f_{2}}^{\perp}\left(t_{1}\right) \rightarrow P_{\rightarrow f_{1}}\left(t_{2}\right)
$$

does not correspond to the pair of decays $f_{2}$ at $t_{1}, f_{1}$ at $t_{2}>t_{1}$, neither in the initial nor in the final decays. This is the "orthogonality problem" [9] that prevent taking an arbitrary pair of decay channels. To connect the $\mathrm{T}$ transformed transition with experiment, we need to find a pair of decay channels such that, for each of them

$$
\text { Given } f \rightarrow \exists f^{\prime} /\left|P_{\rightarrow f}\right\rangle=\left|P_{\rightarrow f}^{\perp}\right\rangle
$$

This condition is satisfied by either $\mathrm{CP}$ conjugate decay channels $\left(P^{0}, \bar{P}^{0}\right)$ or $\mathrm{CP}$ eigenstates of opposite sign with the same flavour content $\left(P_{+}, P_{-}\right)$and no direct $\mathrm{CP}$ violation. Hence the exceptionality of the transitions between semileptonic and $\mathrm{CP}$ eigenstate decays. The method has also been applied to $K^{0}-\bar{K}^{0}$ at a $\Phi$-factory [10].

As a consequence, the orthogonality condition limits the pair of decay channels suitable for $T$-symmetry tests if we start, as a Reference, from the "experimental" transition $\left(P_{\rightarrow f_{1}}\left(t_{1}\right) \rightarrow P_{\rightarrow f_{2}}^{\perp}\left(t_{2}\right)\right)$. This limitation is the prize to be paid to obtain a genuine $T$ violation asymmetry: given $f$ what is the decay channel $f^{\prime}$ such that the $P$ state not going to $f^{\prime}$ coincides with the orthogonal state to the P state not going to ? [9].

The scientific community was recently interested in extending the T-symmetry tests to additional pairs of decay channels, in a program similar to the one developed for $\mathrm{CP}$ 
violation studies. How to do it ? Having identified the orthogonality problem, we give a bypass consisting in having an alternative reference transition once we fix the two channels $f_{1}, f_{2}$. We make the following replacements

\begin{tabular}{|c|c|c|c|}
\hline & BABAR & & NEW \\
\hline $\begin{array}{c}\text { REFERENCE } \\
\text { TRANSITION }\end{array}$ & $P_{\rightarrow f_{1}}\left(t_{1}\right) \rightarrow P_{\rightarrow f_{2}}^{\perp}\left(t_{2}\right)$ & $\Rightarrow$ & $P_{\rightarrow f_{1}}\left(t_{1}\right) \rightarrow P_{\rightarrow f_{2}}\left(t_{2}\right)$ \\
\hline $\begin{array}{c}\text { T-TRANSFORMED } \\
\text { TRANSITION }\end{array}$ & $P_{\rightarrow f_{2}}^{\perp}\left(t_{1}\right) \rightarrow P_{\rightarrow f_{1}}\left(t_{2}\right)$ & $\Rightarrow$ & $P_{\rightarrow f_{2}}\left(t_{1}\right) \rightarrow P_{\rightarrow f_{1}}\left(t_{2}\right)$ \\
\hline
\end{tabular}

Whereas now the two initial $P$-states are directly connected to experiment, the price to be paid is that the two final states are not. One has to work out what is the connection of the novel genuine theoretical T-asymmetry observable to experimental measurements.

\section{The new T violating asymmetry}

As stated, the novel asymmetry proposed for a T-symmetry test is then [9]

$$
A\left(f_{1}, f_{2} ; t\right) \equiv \frac{\operatorname{Pr}\left[P_{\rightarrow f_{1}} \rightarrow P_{\rightarrow f_{2}}(t)\right]-\operatorname{Pr}\left[P_{\rightarrow f_{2}} \rightarrow P_{\rightarrow f_{1}}(t)\right]}{\operatorname{Pr}\left[P_{\nrightarrow f_{1}} \rightarrow P_{\nrightarrow f_{2}}(t)\right]+\operatorname{Pr}\left[P_{\nrightarrow f_{2}} \rightarrow P_{\rightarrow f_{1}}(t)\right]}
$$

where $\operatorname{Pr}\left[P_{\rightarrow f_{1}} \rightarrow P_{\rightarrow f_{2}}(t)\right]$ is the probability that an initially prepared $\left|P_{\rightarrow f_{1}}\right\rangle$ becomes after a time $t$ an $\left|P_{\rightarrow f_{2}}\right\rangle$ state. If $\left|P_{\rightarrow f_{1}}(t)\right\rangle$ is the evolved state at time $t$ of a $\left|P_{\rightarrow f_{1}}\right\rangle$ at time $t=0$, then this probability will be:

$$
\operatorname{Pr}\left[P_{\nrightarrow f_{1}} \rightarrow P_{\nrightarrow f_{2}}(t)\right]=\left|\left\langle P_{\rightarrow f_{2}} \mid P_{\rightarrow f_{1}}(t)\right\rangle\right|^{2}
$$

This probability can be rewritten [9], using closure in the two-dimensional space of the meson system as

$$
\begin{aligned}
\operatorname{Pr}\left[P_{\rightarrow f_{1}}\right. & \left.\rightarrow P_{\rightarrow f_{2}}(t)\right]=\left\langle P_{\rightarrow f_{1}}(t) \mid P_{\rightarrow f_{2}}\right\rangle\left\langle P_{\rightarrow f_{2}} \mid P_{\rightarrow f_{1}}(t)\right\rangle= \\
& =\left\langle P_{\nrightarrow f_{1}}(t)\left|\left[I-\left|P_{\nrightarrow f_{2}}^{\perp}\right\rangle\left\langle P_{\nrightarrow f_{2}}^{\perp}\right|\right]\right| P_{\nrightarrow f_{1}}(t)\right\rangle= \\
& =\left\langle P_{\rightarrow f_{1}}(t) \mid P_{\rightarrow f_{1}}(t)\right\rangle-\left|\left\langle P_{\rightarrow f_{2}}^{\perp} \mid P_{\rightarrow f_{1}}(t)\right\rangle\right|^{2}
\end{aligned}
$$

with the second term directly connected to the probability for the decay of a tagged $\left|P_{\rightarrow f_{1}}\right\rangle$ state at $t_{1}$ to $f_{2}$ after a time $t=t_{2}-t_{1}$ using the "filtering identity" equation (8)

$$
\operatorname{Pr}\left[P_{\rightarrow f_{1}} \rightarrow P_{\rightarrow f_{2}}(t)\right]=\left\langle P_{\rightarrow f_{1}}(t) \mid P_{\rightarrow f_{1}}(t)\right\rangle-\frac{\left|\left\langle f_{2}|W| P_{\rightarrow f_{1}}(t)\right\rangle\right|^{2}}{\left(\left|A_{f_{2}}\right|^{2}+\left|\bar{A}_{f_{2}}\right|^{2}\right)}
$$

Note that also the first term in the right-hand-side of equation (16) is a well defined and measurable quantity: the ratio between the number of mesons that have not decayed after a time $t$ and the initial number tagged at $t_{1}$ as $\left|P_{\rightarrow f_{1}}\right\rangle$ by the observation of the first decay $f_{1}$. We may then call this term the "total survival probability". The other piece in equation (16) is the "normalized" usual double rate at a meson factory with the decaying 
states $f_{1}$ and $f_{2}$ ordered in time. Similarly, for the T transformed transition associated to the Motion Reversal Asymmetry. Therefore we emphasize that this new observable becomes entirely measurable in a meson factory for any pair of decay channels $f_{1}, f_{2}$.

We have not imposed any particular condition to the pair of decay channels $f_{1}, f_{2}$, so one is not forced to use flavour specific or $C P$ eigenstate decay channels. We will discuss later whether the measurable $A\left(f_{1}, f_{2} ; t\right)$ becomes a genuine Time-Reversal- Violation Asymmetry for any pair of decays .

\section{The Theoretical Expressions}

Using the time evolution imposed by Quantum Mechanics we know the time dependent structure of the needed measurable quantities in terms of $\Delta m, \Gamma$ and $\Delta \Gamma$ [8], determined by the eigenvalues of the entire Hamiltonian for the $\left(P^{0}, \bar{P}^{0}\right)$ system

$$
\begin{gathered}
\left\langle P_{\rightarrow f}(t) \mid P_{\rightarrow f}(t)\right\rangle=e^{-\Gamma t}\left\{\begin{array}{l}
C_{h}[f] \cosh \left(\frac{\Delta \Gamma t}{2}\right)+C_{c}[f] \cos (\Delta m t) \\
S_{h}[f] \sinh \left(\frac{\Delta \Gamma t}{2}\right)+S_{c}[f] \sin (\Delta m t)
\end{array}\right\} \\
\frac{\left|\left\langle g|W| P_{\rightarrow f}(t)\right\rangle\right|^{2}}{\left(\left|A_{\mathrm{g}}\right|^{2}+\left|\bar{A}_{\mathrm{g}}\right|^{2}\right)}=e^{-\Gamma t}\left\{\begin{array}{l}
\mathbf{C}_{h}[f, g] \cosh \left(\frac{\Delta \Gamma t}{2}\right)+\mathbf{C}_{c}[f, g] \cos (\Delta m t) \\
\mathbf{S}_{h}[f, g] \sinh \left(\frac{\Delta \Gamma t}{2}\right)+\mathbf{S}_{c}[f, g] \sin (\Delta m t)
\end{array}\right\}
\end{gathered}
$$

In other words, each one of the two transitions is determined by the four measurable parameters $C_{h}[f], C_{c}[f], S_{h}[f], S_{c}[f]$ and $\mathbf{C}_{h}[f, g], \mathbf{C}_{c}[f, g], \mathbf{S}_{h}[f, g], \mathbf{S}_{c}[f, g]$.

The numerator of the asymmetry $N(f, g ; t)$

$$
A(f, g ; t)=\frac{\operatorname{Pr}\left[P_{\rightarrow f} \rightarrow P_{\rightarrow g}(t)\right]-\operatorname{Pr}\left[P_{\rightarrow g} \rightarrow P_{\rightarrow f}(t)\right]}{\operatorname{Pr}\left[P_{\rightarrow f f} \rightarrow P_{\rightarrow g}(t)\right]+\operatorname{Pr}\left[P_{\rightarrow g} \rightarrow P_{\rightarrow f}(t)\right]}=\frac{N(f, g ; t)}{D(f, g ; t)}
$$

is then given by three "asymmetry parameters": the non-vanishing value of any of these asymmetry parameters would be a signal of Time-Reversal-Violation.

$$
N(f, g ; t)=e^{-\Gamma t}\left\{\begin{array}{c}
C^{N}[f, g]\left\{\cosh \left(\frac{\Delta \Gamma t}{2}\right)-\cos (\Delta m t)\right\} \\
S_{h}^{N}[f, g] \sinh \left(\frac{\Delta \Gamma t}{2}\right)+S_{c}^{N}[f, g] \sin (\Delta m t)
\end{array}\right\}
$$

The theoretical connection of these measurable parameters to the matrix element of the meson evolution Hamiltonian $H_{i j}$ involved in the Weisskoppf-Wigner Approach (WWA) for the $\left(P^{0}, \bar{P}^{0}\right)$ system is $[11,12,13]$ 


$$
\begin{gathered}
C^{N}[f, g]=\delta\left(C_{f}-C_{g}\right)-\operatorname{Im}(\theta)\left(S_{f}-S_{g}\right) \\
S_{h}^{N}[f, g]=\delta\left(C_{f} R_{g}-C_{g} R_{f}\right)+\operatorname{Im}(\theta)\left(R_{f} S_{g}-R_{g} S_{f}\right) \\
S_{c}^{N}[f, g]=\left(C_{f} S_{g}-C_{g} S_{f}\right)\left\{1+\delta\left(C_{f}+C_{g}\right)\right\}+ \\
+\delta\left(S_{f}-S_{g}\right)+\operatorname{Re}(\theta)\left(R_{f} S_{g}-R_{g} S_{f}\right)
\end{gathered}
$$

where we have presented the results at leading order in terms of the usual mixing parameters -see the book by G. Branco et al. [8]- $\delta=\left(1-|q / p|^{2}\right) /\left(1+|q / p|^{2}\right)$, that is real, $T$ and $C P$ violating, and the complex parameter $\theta$, that is $C P T$ and $C P$ violating, $\theta=\left(H_{22}-H_{11}\right) /(\Delta m-i \Delta \Gamma / 2)$. We also use the parameter $R_{i}, S_{i}$ and $C_{i}$ defined in terms of the well-known $\lambda_{i}=q \bar{A}_{f_{i}} / p A_{f_{i}}$

$$
C_{i}=\frac{\left(1-\left|\lambda_{i}\right|^{2}\right)}{\left(1+\left|\lambda_{i}\right|^{2}\right)} \quad ; \quad S_{i}=\frac{2 \operatorname{Im}\left(\lambda_{i}\right)}{\left(1+\left|\lambda_{i}\right|^{2}\right)} \quad ; \quad R_{i}=\frac{2 \operatorname{Re}\left(\lambda_{i}\right)}{\left(1+\left|\lambda_{i}\right|^{2}\right)}
$$

where $q / p$ in the CPT violating case is defined in the usual way $(q / p)^{2}=H_{21} / H_{12}$. Note that this parameters are not all independent $C_{i}^{2}+S_{i}^{2}+R_{i}^{2}=1$.

In order to show how the new T-violating observable really open the T analysis to all channels at a B-factory we will concentrate in the case where we choose $f$ to be a flavour specific channel corresponding to $\left|P_{\rightarrow f}\right\rangle=\left|\bar{B}^{0}\right\rangle\left(\left|P_{\rightarrow f}\right\rangle=\left|B^{0}\right\rangle\right)$ and $\bar{A}_{f}=0$ $\left(A_{f}=0\right)$ therefore $\lambda_{f}=0\left(\lambda_{f}=\infty\right)$

$$
C_{f}=1\left(C_{f}=-1\right) \quad ; \quad S_{f}=0 ; \quad R_{f}=0
$$

To avoid complications we also use the approximation $\Delta \Gamma=0$ and $\delta=0$. We will use, even if misleading, the notation $f=B^{0}\left(f=\bar{B}^{0}\right)$. Under this conditions we have in any channel $g$ for the numerator of the asymmetry

$$
\begin{gathered}
N\left(B^{0}, g ; t\right)=e^{-\Gamma t} S_{g}\{\sin (\Delta m t)+\operatorname{Im} \theta[1-\cos (\Delta m t)]\} \\
N\left(\bar{B}^{0}, g ; t\right)=e^{-\Gamma t} S_{g}\{-\sin (\Delta m t)+\operatorname{Im} \theta[1-\cos (\Delta m t)]\}
\end{gathered}
$$

For completeness we also give the denominator

$$
\begin{aligned}
& D\left(B^{0}, g ; t\right)=e^{-\Gamma t}\left\{\begin{array}{c}
\left(1+C_{g} \cos (\Delta m t)\right)-R_{g} \operatorname{Re} \theta(1-\cos (\Delta m t)) \\
+\operatorname{Im} \theta\left(1+C_{g}\right) \sin (\Delta m t)
\end{array}\right\} \\
& D\left(\bar{B}^{0}, g ; t\right)=e^{-\Gamma t}\left\{\begin{array}{c}
\left(1-C_{g} \cos (\Delta m t)\right)+R_{g} \operatorname{Re} \theta(1-\cos (\Delta m t)) \\
-\operatorname{Im} \theta\left(1-C_{g}\right) \sin (\Delta m t)
\end{array}\right\}
\end{aligned}
$$

Note that for $g=J / \psi K_{S}^{0}$ we get an asymmetry of the same order than the one measured by BABAR.

$$
A\left(B^{0}, J / \psi K_{S}^{0} ; t\right) \sim S_{J / \psi K_{S}^{0}} \sin (\Delta m t)=\sin (2 \beta) \sin (\Delta m t)
$$


But of course experimentally is completely different: here there is no $J / \psi K_{L}^{0}$. It is interesting to note also

$$
\left\langle P_{\rightarrow f}(t) \mid P_{\rightarrow f}(t)\right\rangle=e^{-\Gamma t}\left\{1+O(\delta)+O(\theta)+R_{f} O\left(\frac{\Delta \Gamma t}{2}\right)\right\} \sim e^{-\Gamma t}
$$

\section{Selecting a few channels}

A superficial inspection of the numerator of our asymmetry -see equations $(20,21,24)$ would indicate that the existence of a non-vanishing factor $S_{g}$ is, by itself, a signal of $T$ violation. This conclusion would be wrong in general. The problem can be traced back to antiunitary character of the operator $U_{T}$ implementing the $T$ symmetry in the space of physical states. The principle of $T$-invariance or microreversibility strictly means [14] that

$$
\left|\left\langle U_{T} P_{\nrightarrow f}|U(t, 0)| U_{T} P_{\nrightarrow g}\right\rangle\right|^{2}=\left|\left\langle P_{\nrightarrow g}|U(t, 0)| P_{\nrightarrow f}\right\rangle\right|^{2}
$$

But not in general

$$
\left|\left\langle P_{\rightarrow f}|U(t, 0)| P_{\rightarrow g}\right\rangle\right|^{2}=\left|\left\langle P_{\nrightarrow g}|U(t, 0)| P_{\rightarrow f}\right\rangle\right|^{2}
$$

being $U_{T}=\widehat{U}_{T} K$ where $K$ is the complex conjugation operator and $\widehat{U}_{T}$ is a unitary operator almost irrelevant in the meson case. The possible difficulty in assigning a genuine character of $T$ violation to the numerator of our asymmetry (19) is concentrated on $K$. Sufficient conditions to satisfy

$$
U_{T}\left|P_{\rightarrow f}\right\rangle=e^{i \phi}\left|P_{\rightarrow f}\right\rangle
$$

in the $T$ invariant limit will be presented in a future publication. Here we mention an incomplete list of types of decay channels that can be used with the effect of $U_{T}$ being a global phase factor:

1) Flavour Specific (FS) channels.

2) $\mathrm{CP}$ eigenstates without direct $\mathrm{CP}$ violation. The absence of direct $\mathrm{CP}$ violation in the decay to $\mathrm{CP}$ eigenstates makes irrelevant the presence of the complex conjugation operator, in the sense that it changes the global phase of $\left|P_{\rightarrow f}\right\rangle$ and therefore $S_{g} \neq 0$ is a genuine signal of $T$ violation.

3) For arbitrary states, including non $\mathrm{CP}$ eigenstates, without $\mathrm{CP}$ violation in the decay one is able to prove

$$
\lambda_{g} \lambda_{\bar{g}}=e^{-2 i \xi}\left(\frac{q}{p}\right)^{2}
$$

where $\xi$ is the arbitrary phase of the CP operator $U_{C P}\left|P^{0}\right\rangle=e^{i \xi}\left|\bar{P}^{0}\right\rangle$ in such a way that $\lambda_{g} \lambda_{\bar{g}} \neq 1$ is signal of $\mathrm{T}$ violation. Note that in the $\mathrm{T}$ invariant limit $(q / p)^{2}=e^{+2 i \xi}[8]$. As a consequence the genuine signal of $\mathrm{T}$ violation is given by the condition $S_{g}+S_{\bar{g}} \neq 0$. 
4) A decay product that is an eigenstates of the Strong Scattering Matrix. One case of possible interest is that of the $K^{0}, \bar{K}^{0} \rightarrow(\pi \pi)_{I}$ channels with welldefined Isospin $I$ at a $\Phi$-factory.

It is worth noticing that the asymmetries constructed with the combination of semileptonic and CP eigenstate decay channels are now experimentally different from those already measured by BABAR Collaboration. A case of particular interest is given by the reference transition $B^{0}$ or $\bar{B}^{0}$, leading to $J / \psi K_{S}^{0}$ which does not involve in the novel asymmetry $J / \psi K_{L}^{0}$. This case can be extended to other charmonium final states and many more channels like $\varphi K_{S}^{0}, \pi^{0} K_{S}^{0}, \rho^{0} K_{S}^{0}, J / \psi \pi^{0}$ and $(\pi \pi)_{I=2}$ as some interesting examples.

For the decay products which are not $\mathrm{CP}$ eigenstates, our results suggest the use of $N\left(B^{0}, g ; t\right)$ and $N\left(\bar{B}^{0}, g ; t\right)$ which can be combined to avoid any possible presence of fake $T$ violation in the individual transitions. The following observable:

$$
\begin{gathered}
N\left(B^{0}, g ; t\right)+N\left(B^{0}, \bar{g} ; t\right)= \\
=e^{-\Gamma t}\left(S_{g}+S_{\bar{g}}\right)\{\sin (\Delta m t)+\operatorname{Im} \theta[1-\cos (\Delta m t)]\}
\end{gathered}
$$

for decay channels $g, \bar{g}$ without direct CP violation, is a genuine $T$ violating observable. Among those channels we have, for example, $g=D^{*}(2010)^{+} D^{-}, \bar{g}=D^{*}(2010)^{-} D^{+}$ where this asymmetry should be almost maximal: $\left(S_{g}+S_{\bar{g}}\right)=2 *(-0.73 \pm$ 0.11 ) [15]. Other channels like $D^{* \pm} \pi^{\mp}$ or even in $B_{S}^{0} \rightarrow D_{S}^{ \pm} K^{\mp}$ should be of interest.

A final remark concerns the fact that we can combine several asymmetry numerators to avoid the measurement of the total survival probabilities:

$$
N\left(f_{1}, f_{2}\right)+N\left(f_{2}, f_{3}\right)+N\left(f_{3}, f_{1}\right)
$$

\section{Conclusions}

We have modified the reference transition of BABAR $T$ violating measurement $P_{\rightarrow f_{1}}\left(t_{1}\right) \rightarrow P_{\rightarrow f_{2}}^{\perp}\left(t_{2}\right)$ - directly connected to the decay $Y(4 S) \rightarrow f_{1}\left(t_{1}\right)+f_{2}\left(t_{2}\right)$ - by $P_{\rightarrow f_{1}}\left(t_{1}\right) \rightarrow P_{\rightarrow f_{2}}\left(t_{2}\right)$. With the New Reference we proposed in addition to the Babar and CPLEAR asymmetries the asymmetry in equation (19). The connection to the experimental observables is given by the survival probability defined and calculated in equation (17) and the normalized double decay rate to $f_{1}$ at $t_{1}$ and $f_{2}$ a later time $t_{2}=t+t_{1}$ introduced in the second term of the r.h.s. of equation (16).

The proposed asymmetry can be used for decay products that include flavour specific, $C P$ eigenstates and non $C P$ eigenstates. In the last case one has to combine the reference transitions $B^{0} \rightarrow g$ and $B^{0} \rightarrow \bar{g}$. In this way we eliminate possible sources of fake $T$ violation. With the proposal here discussed, the way is more open to a full experimental program [16] of studies of $T$ violation observables at meson factories.

\section{Acknowledgements}

FJB would like to acknowledge George Zoupanos for the excelent atmosphere we had at Corfu Summer Institute in September 2013. This work has been supported by Spanish 
MINECO (FEDER) under grant FPA2011-23596 and by Generalitat Valenciana under grants PROMETEOII 2014-049 and PROMETEOII 2013-017.

\section{References}

[1] Lees J, et al., Observation of Time Reversal Violation in the B0 Meson System, Phys.Rev.Lett. 109 (2012) 211801 (arXiv:1207.5832).

[2] Bernabeu J, Martinez-Vidal F, Villanueva-Perez P, Time Reversal Violation from the entangled B0antiB0 system, J. High Energy Phys. JHEP 1208 (2012) 064 (arXiv:1203.0171).

[3] Banuls M, Bernabeu J, CP, T and CPT versus temporal asymmetries for entangled states of the B(d) system, Phys.Lett. B464 (1999) 117-122 (hep-ph/9908353).

[4] Banuls M, Bernabeu J, Studying indirect violation of CP, T and CPT in a B factory, Nucl.Phys. B590 (2000) 19-36 (hep-ph/0005323).

[5] Kabir P, What is not invariant under time reversal?, Phys.Rev. D2 (1970) 540-542.

[6] Angelopoulos A et al., First direct observation of time reversal noninvariance in the neutral kaon system, Phys.Lett. B444 (1998) 43-51.

[7] Lipkin H J, Simple symmetries in EPR correlated decays of kaon and B meson pairs with CP violation, Phys.Lett. B219 (1989) 474

[8] Branco G C, Lavoura L, Silva J P, CP Violation, Inter. Ser. Monogr. Phys vol. 103, Oxford University Press, 1999.

[9] Bernabeu J, Botella F J, Nebot M, Novel T -Violation observable open to any pair of decay channels at meson factories, Phys.Lett. B728 (2014) 95-98 (arXiv:1309.0439).

[10] Bernabeu J, Di Domenico A, Villanueva-Perez P, Direct test of time reversal symmetry in the entangled neutral kaon system at a Phi-factory, Nucl.Phys. B868 (2013) 102-119, (arXiv: 1208.0773)

[11] Weisskopf V, Wigner E P, Calculation of the natural brightness of spectral lines on the basis of Dirac's theory, Z.Phys. 63 (1930) 54-73.

[12] Weisskopf V, Wigner, Over the natural line width in the radiation of the harmonius oscillator, Z.Phys. 65 (1930) 18-29.

[13] Lee T D, Oehme R, Yang C N, Remarks on Possible Noninvariance Under Time Reversal and Charge Conjugation, Phys.Rev. 106 (1957) 340-345.

[14] Sachs R G, The Physics of Time Reversal, The University of Chicago Press, 1-325(1987)

[15] Y. Amhis, et al., Heavy Flavor Averaging Group, Averages of b-hadron, c-hadron and $\tau$ lepton properties as of early 2012, arXiv:1207.1158.

[16] A. Bevan, G. Inguglia, M. Zoccali, arXiv:1302.4191. 